\title{
Role of Diffusion-Weighted Magnetic Resonance Imaging in Laryngeal and Hypopharyngeal Cancer in Comparison to Histopathological Finding
}

\author{
Essam A. Behairy ${ }^{1}$, Naser M. Abd El-Bary ${ }^{2}$, Mohammed E. Abd El-Samea ${ }^{3}$, \\ Hagar A. Abdel-Muttalib*1, Ahmad M. Hamadan ${ }^{1}$
}

Departments of ${ }^{1}$ Otorhinolaryngology and ${ }^{2}$ Clinical Oncology, Faculty of Medicine, ${ }^{3}$ Department of Diagnostic Medical and Interventional Radiology, National liver Institute, Menoufia University, Egypt

*Corresponding Author: Hagar Ahmed Mohammed Abdel-Muttalib, Mobile: 00201011599909,

Email: grgrhama511@gmail.com

\begin{abstract}
Background: Diffusion-weighted magnetic resonance imaging (DW-MRI) has the ability of tissue characterization and can differentiate between benign and malignant tissues, including differentiation between benign postradiotherapy changes and residual or recurrent malignancy.

Objective: This study aimed to assess the role of diffusion-weighted magnetic resonance imaging in the evaluation of laryngeal and hypopharyngeal cancer as an initial diagnosis and the post-treatment surveillance protocol.

Patients and methods: This study was a cross-sectional analytical study including twenty-five patients who presented with laryngeal or hypopharyngeal masses, thirteen cases were pretreatment cases, and twelve cases were posttreatment cases after completion of treatment. Seven cases had a hypopharyngeal origin, and eighteen cases had a laryngeal origin. The patients were assessed using history taking, a telescopic examination of the larynx and hypopharynx, computed tomography scanning, and diffusion-weighted magnetic resonance imaging on the neck. Direct laryngoscopic examination and biopsy taking from suspicious lesions were done. Biopsies were subjected to histopathological examination with a correlation between pathological and DW- MRI radiological results.

Results: DW-MRI had a 100\% sensitivity, $82.4 \%$ specificity, and $88 \%$ accuracy compared to histopathological findings of biopsies from the studied group. There was a non-significant difference between DW-MRI and biopsy findings for either pretreatment or post-treatment cases of both laryngeal and hypopharyngeal origin of the lesions ( $\mathrm{p}$ value $>0.05$ for all).
\end{abstract}

Conclusion: DW-MRI is a sensitive tool for detecting laryngeal and hypopharyngeal cancers in new cases and excluding tumor residual after chemo-radiotherapy in cases with suspicious lesions after completion of treatment.

Keywords: Apparent Diffusion Coefficient, Chemotherapy, Diffusion-Weighted MRI, Laryngeal cancer, Hypopharyngeal cancer, Radiotherapy.

\section{INTRODUCTION}

DW-MRI has been introduced to evaluate head and neck cancers ${ }^{(\mathbf{1})}$. It is based on the relative mobility of water molecules in different tissues and provides information about the microenvironment, such as cell density, cell integrity, and vascularity ${ }^{(2)}$. These water mobility differences are quantified by using the apparent diffusion coefficient (ADC) ${ }^{(3)}$. Measurement of ADCs calculated from DW-MRI may be used to characterize laryngeal and hypopharyngeal lesions and early detection of their malignancy (4). Diffusionweighted imaging (DWI) quantified by (ADC) can also help in the early detection of residual laryngeal and hypopharyngeal tumors after treatment with chemoradiotherapy (CRT) as it allows differentiation of postCRT inflammation and necrosis (treatment-induced tissue changes) from recurrent malignancy. The historadiological correlation has shown an inverse correlation between the ADC and lesion cellularity ${ }^{(5)}$.

The most reliable method of detecting recurrent tumor is direct laryngoscopy under general anesthesia. Biopsies from suspicious areas are taken for histopathological examination ${ }^{(6)}$. However, this method is not always accurate or available as it can miss recurrent tumors directly under the mucosa or wrongly assess the multifocal nature of the tumor. A negative biopsy is not sufficient to exclude tumors, and sometimes biopsy taking is risky in some patients who are unfit for interference. Therefore, a non-invasive imaging tool could be used to select patients who might benefit from a laryngoscopy, thereby limiting unnecessary biopsies and patient morbidity, and may even indicate the most appropriate biopsy location ${ }^{(2)}$.

Correlation between the non-invasive method (DW-MRI) and the invasive method as the direct laryngoscopic biopsy is of great benefit in detecting recurrence and residual malignancy ${ }^{(2,7)}$. This study aimed to assess the role of DW-MRI in the evaluation of laryngeal and hypopharyngeal cancer as an initial diagnosis and the post-treatment surveillance protocol.

\section{PATIENTS AND METHODS}

The current study was a cross-sectional analytical study evaluating the role of DW-MRI in laryngeal and hypopharyngeal cancer compared to histopathological findings. The patients of this study were recruited from the Otorhinolaryngology and

This article is an open access article distributed under the terms and conditions of the Creative Commons Attribution (CC BY-SA) license (http://creativecommons.org/licenses/by/4.0/) 
Oncology Departments, (removed for blinding) during the period from June 2019 to June 2020.

The sample size was calculated based on a previous study by Vandecaveye et al. ${ }^{(7)}$, which showed the difference between signal intensity between head and neck squamous cell carcinoma and non-tumoral post-radiotherapy tissues at b1000 was 123 a.u. with a standard deviation of 84 a.u. The total sample size was calculated to be 15 to achieve power of $90 \%$ and 0.05 alpha I error, including 30\% compensation for the loss in follow up.

The current study included patients with laryngeal or hypopharyngeal masses as detected by telescopic examination of the larynx and the hypopharynx to assess alarming symptoms as hoarseness of voice more than two weeks with risk factors like smoking, any degree of airway obstruction, or difficulty of swallowing. The study also included patients who had received chemotherapy or radiotherapy or combined chemo-radiotherapy as a part of post-treatment surveillance protocol. Assessments of these patients were three months after completion of their treatment regimen. Any patient with a history of cardiac pacemakers, cochlear implants were excluded from the study. Also, patients with hypersensitivity to gadolinium were excluded from the study.

Patients were subjected to an assessment protocol including history taking to detect new-onset of alarming symptoms like hoarseness of voice, airway obstruction, difficult swallowing, or worsening of symptoms after chemo/radiotherapy in patients previously diagnosed as having laryngeal or hypopharyngeal carcinomas. Complete otorhinolaryngology examination was performed for every patient, including telescopic examination of the larynx and hypopharynx to verify any abnormality as masses or any change of the previous examination of the same patient, and neck examination for enlarged cervical lymph nodes.

The study included twenty-five patients, including thirteen pretreatment cases and twelve posttreatment cases after completion of treatment. Seven cases had a hypopharyngeal origin, and eighteen cases had a laryngeal origin.

Patients were subjected to computed tomography scanning to detect the presence of any anatomical abnormality, including its specific site, size, delineation, relations to surrounding structures, and presence of suspicious lymph nodes. MRI with diffusion on the neck with emphasis on the larynx, hypopharynx was done. All patients were scanned at the radiology department, (Removed for blinding) on the same GE MRI SCANNER Optima ${ }^{\mathrm{TM}}$ MR450wGEM-70cm (General electric company, Boston, USA). DW-MRI was performed as Axial T2 fat sat, Diffusion WIs with b values 0, 500, and 1000 . Apparent Diffusion Coefficient (ADC) was calculated as a measurement for the diffusion of water molecules in the tissues with the cutoff value calculated as $1.5 \times 10^{-}$ ${ }^{3} \mathrm{~mm}^{2}$ above, which is facilitated diffusion, and below, which is restricted diffusion.

Patients of the study were subjected to direct laryngoscopic examination of the larynx and hypopharynx under general anesthesia with biopsy taking from suspicious lesions. Histopathological examination of biopsies was done with a correlation between radiological results of DW-MRI and pathological results.

\section{Ethical approval:}

An approval of the study was obtained from Menoufia University academic and ethical committee. Every patient signed an informed written consent for acceptance of the operation.

Statistical analysis: Data were collected, tabulated, and statistically analyzed by an IBM compatible personal computer with SPSS statistical package version 23 (IBM Corp., Armonk, NY, US). Descriptive statistics included qualitative data that were expressed as number (No.) and percentage (\%). Quantitative data were expressed as mean (X), standard deviation (SD), median, and range. Analytic statistics included a Chisquare test $(\chi 2)$ to study the association between qualitative variables. Whenever any of the expected cells were less than five, Fischer's Exact test was used. A P-value of $<0.05$ was considered statistically significant. P-value of $<0.001$ was considered statistically highly significant.

\section{RESULTS}

The current study included twenty-five patients distributed as eighteen males (72\%) and seven females (28\%) with an age ranging from 35 to 79 years old and mean age of $54.4 \pm 12.5$. Ten of the patients were smokers, seven were ex-smokers, and only two were shisha smokers. Thirteen cases $(52 \%)$ were pretreatment, while twelve cases $(48 \%)$ were posttreatment cases after chemoradiation. Seven cases (28\%) had a hypopharyngeal origin, and eighteen cases (72\%) had a laryngeal origin. Twelve patients (48\%) presented with hoarseness of voice, eight patients (32\%) with difficulty in breathing up to stridor, five patients (20\%) with dysphagia, and five patients (20\%) with an enlarged cervical lymph node (Table 1).

Table (1): Socio-demographic and clinical characteristics of the studied group. 


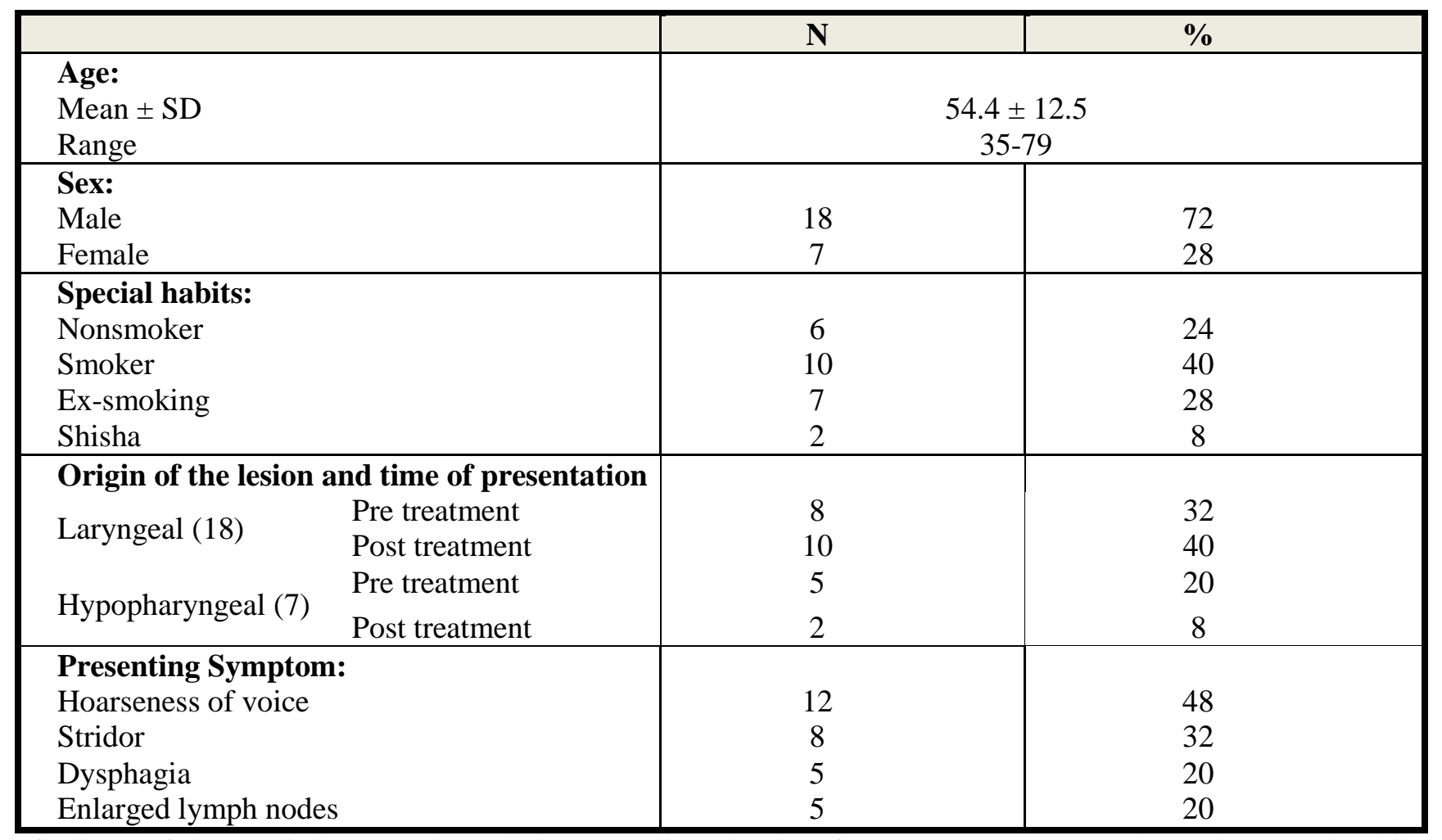

MRI findings of the studied group showed that regarding the site: four cases (16\%) were hypopharyngeal, while sixteen cases $(64 \%)$ were laryngeal, including three (12\%) supraglottic, three (12\%) transglottic, eight (32\%) glottic and two (8\%) subglottic case. Five cases of the study patients $(20 \%)$ showed no lesion. Regarding diffusability, the ADC range of the studied group was $0.7-2.25 \times 10-3 \mathrm{~mm}^{2} / \mathrm{sec}$, with the cutoff value being $1.5 \times 10-3 \mathrm{~mm}^{2} / \mathrm{sec}$. Thirteen cases $(52 \%)$ had ADC more than the cutoff value reflecting facilitated diffusion and were considered negative for malignancy. Twelve cases (48\%) had ADC less than the cutoff value reflecting restricted diffusion and were considered positive for malignancy (Table $2 \&$ Figures 1,2).

Table (2): Magnetic resonance imaging findings of the studied group

\begin{tabular}{|c|c|c|}
\hline \multirow{2}{*}{ MRI findings } & \multicolumn{2}{|c|}{ Frequency $(n=25)$} \\
\hline & $\mathbf{N}$ & $\%$ \\
\hline \multicolumn{3}{|l|}{ Radiological site: } \\
\hline Transglottic mass & 3 & 12 \\
\hline Supraglottic mass & 3 & 12 \\
\hline Glottic mass & 8 & 32 \\
\hline Subglottic & 2 & 8 \\
\hline Hypopharyngeal mass & 4 & 16 \\
\hline No lesion & 5 & 20 \\
\hline \multicolumn{3}{|l|}{ Diffusability: } \\
\hline Facilitated (-ve for malignancy) & 13 & 52 \\
\hline Restricted (+ve for malignancy) & 12 & 48 \\
\hline \multicolumn{3}{|l|}{ ADC: } \\
\hline Cut off point & \multicolumn{2}{|c|}{$\begin{array}{c}1.5 \times 10^{-3} \mathrm{~mm}^{2} \\
0.7-2.25\end{array}$} \\
\hline Range & & \\
\hline \multicolumn{3}{|l|}{ ADC grouping: } \\
\hline$\leq 1.5$ & 12 & 48 \\
\hline$>1.5$ & 13 & 52 \\
\hline
\end{tabular}

MRI: Magnetic Resonance Imaging, DW- MRI: Diffusion-Weighted Magnetic Resonance Imaging, ADC: Apparent Diffusion Coefficient 


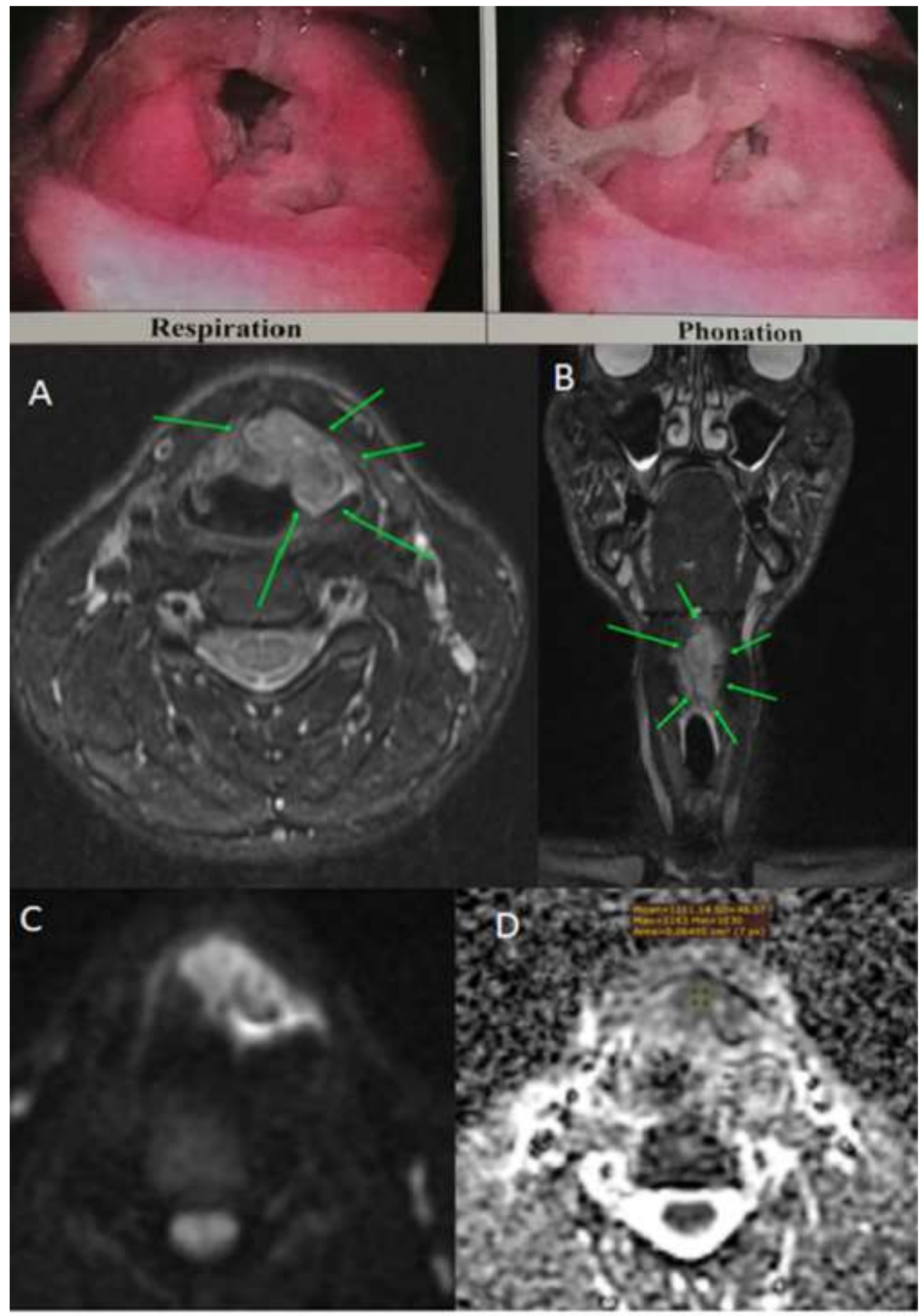

Figure (1): A case of transglottic mass with anterior midline extension involving both vocal folds and measuring 2 $\mathrm{cm}$ x $2.7 \mathrm{~cm}$ x $3.4 \mathrm{~cm}$. Image (A) showed T2 axial Short-TI Inversion Recovery (STIR), image (B) showed T2 coronal STIR, image (C) showed axial DWI b1000 with diffusion. 

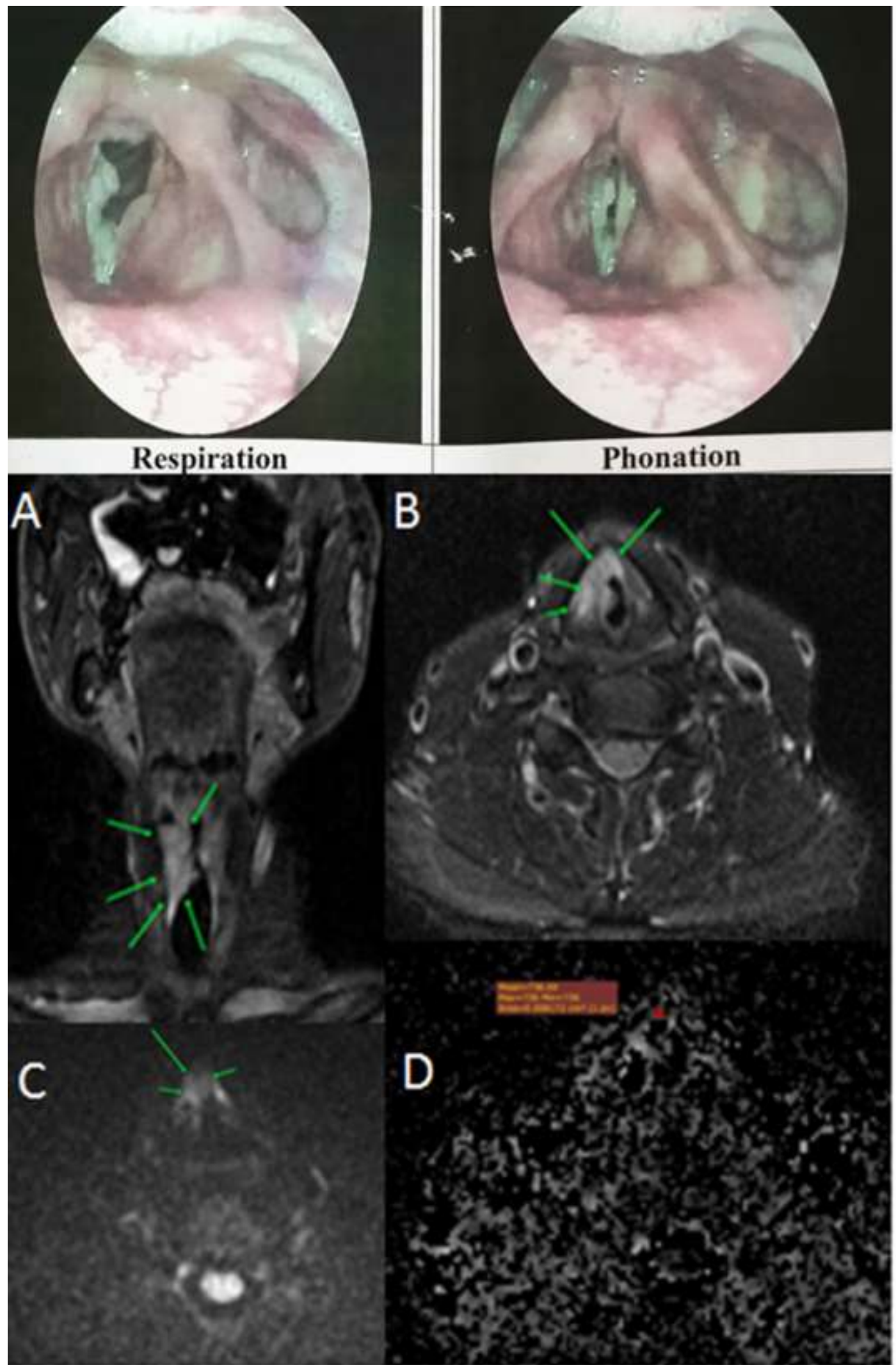

Figure (2): A case of right vocal fold mass with subglottic and anterior commissure extension measuring $14 \mathrm{~mm} \mathrm{x}$ $10 \mathrm{~mm}$ x $20 \mathrm{~mm}$. Image (A) showed T2 coronal Short-TI Inversion Recovery (STIR), image (B) showed T2 axial STIR, image (C) showed axial DWI b1000 with diffusion restriction, and image (D) shows ADC value measuring $0.86 \times 10-3 \mathrm{~mm} 2 / \mathrm{sec}$.

DW-MRI had 100\% sensitivity, $82.4 \%$ specificity, and $88 \%$ accuracy compared to histopathological findings of biopsies from the studied group (Table 3 ). 
Table (3): Evaluation of diffusion-weighted MRI in laryngeal and hypopharyngeal cancer in comparison to histopathological finding

\begin{tabular}{|c|c|c|c|c|c|}
\hline \multirow{3}{*}{\multicolumn{2}{|c|}{ Item }} & \multicolumn{4}{|c|}{ Biopsy (gold standard) } \\
\hline & & \multicolumn{2}{|c|}{ Positive $(n=8)$} & \multicolumn{2}{|c|}{ Negative $(n=17)$} \\
\hline & & $\mathbf{N}$ & $\%$ & $\mathbf{N}$ & $\%$ \\
\hline \multirow[t]{2}{*}{ DW-MRI } & Positive & 8 & 100 & 3 & 17.6 \\
\hline & Negative & 0 & 0 & 14 & 82.4 \\
\hline \multicolumn{2}{|l|}{ Sensitivity } & \multicolumn{4}{|c|}{$100 \%$} \\
\hline \multicolumn{2}{|l|}{ Specificity } & \multicolumn{4}{|c|}{$82.4 \%$} \\
\hline \multicolumn{2}{|l|}{ Accuracy } & \multicolumn{4}{|c|}{$88 \%$} \\
\hline
\end{tabular}

MRI: Magnetic Resonance Imaging, DW- MRI: Diffusion-Weighted Magnetic Resonance Imaging,

All pathologically positive cases for malignancy showed squamous cell carcinoma with various grades of differentiation. There was a non-significant difference between DW-MRI and biopsy findings for either pretreatment and post-treatment cases of both laryngeal and hypopharyngeal origin of the lesions ( $p>0.05$ for all) (Table 4).

Table (4): Comparison between DW-MRI and biopsy findings for either pretreatment and post-treatment cases of both laryngeal and hypopharyngeal origin of the lesions

\begin{tabular}{|c|c|c|c|c|c|c|}
\hline \multirow{2}{*}{\multicolumn{2}{|c|}{ Item }} & \multicolumn{2}{|c|}{ DW-MRI } & \multicolumn{2}{|c|}{ Biopsy } & \multirow{2}{*}{\begin{tabular}{|c} 
Fischer Exact test \\
P value
\end{tabular}} \\
\hline & & No. & $\%$ & No. & $\%$ & \\
\hline \multicolumn{7}{|c|}{ Laryngeal origin (18) } \\
\hline \multirow[t]{2}{*}{ Pretreatment (8) } & $+\mathrm{ve}$ & 6 & 75 & 5 & 62.5 & \multirow{2}{*}{1} \\
\hline & -ve & 2 & 25 & 3 & 37.5 & \\
\hline \multirow[t]{2}{*}{ Post treatment (10) } & $+\mathrm{ve}$ & 0 & 0 & 0 & 0 & \multirow{2}{*}{1} \\
\hline & -ve & 10 & 100 & 10 & 100 & \\
\hline \multicolumn{7}{|c|}{ Hypopharyngeal origin (7) } \\
\hline \multirow[t]{2}{*}{ Pretreatment (5) } & $+\mathrm{ve}$ & 5 & 100 & 3 & 60 & \multirow{2}{*}{0.44} \\
\hline & -ve & 0 & 0 & 2 & 40 & \\
\hline \multirow[t]{2}{*}{ Post treatment (2) } & $+\mathrm{ve}$ & 0 & 0 & 0 & 0 & \multirow{2}{*}{1} \\
\hline & & 2 & 100 & 2 & 100 & \\
\hline
\end{tabular}

DW- MRI: Diffusion-Weighted Magnetic Resonance Imaging

\section{DISCUSSION}

In the current study, we assessed the role of MRI with diffusion as a diagnostic modality for primary, residual and recurrent cases of hypopharyngeal and laryngeal cancers compared to endoscopy and histopathological examination being the gold standard diagnostic modality for any malignancy of the aerodigestive tract. The study included thirteen cases of tumors with primary presentation and twelve cases for follow up biopsy. The study included seven cases of hypopharyngeal origin and eighteen cases of laryngeal origin. We found that MRI with diffusion had a $100 \%$ sensitivity, $82.4 \%$ specificity, and $88 \%$ accuracy compared to histopathological findings of biopsies from the studied group. There was a non-significant difference between DW-MRI and biopsy findings for either pretreatment and post-treatment cases of both laryngeal and hypopharyngeal origin of the lesions ( $p$ > 0.05 for all)

Several studies in the literature assessed the role of MRI with diffusion in evaluating post-treatment cases of head and neck carcinoma, with few studies evaluating its role as a diagnostic modality. These studies' results match with the results of our study and support the role of MRI with diffusion in head and neck cancer. Wang et al. ${ }^{\text {(4) }}$ stated that measurement of ADCs calculated from diffusion-weighted echo-planar magnetic resonance images may be used to characterize head and neck lesions with an accuracy of $86 \%$, with $84 \%$ sensitivity and $91 \%$ specificity. Vandecaveye et al. ${ }^{(8)}$ found that the differentiation of tumoral tissue from radiotherapy-induced tissue alterations was possible with DW-MRI. Vandecaveye et $\boldsymbol{a l} .{ }^{(7)}$ stated that DWMRI accurately differentiated persistent or recurrent head and neck squamous cell carcinoma from nontumoral tissue changes after (chemo) radiotherapy with a sensitivity of $94.6 \%$, a specificity of $95.9 \%$, and an accuracy of $95.5 \%$.

Abdel Razek et al. ${ }^{(9)}$ stated that Diffusionweighted MR imaging with ADC measurement had promising results for differentiating residual or recurrent head and neck tumors from postoperative or post-radiation changes with an accuracy of $87 \%$, a sensitivity of $84 \%$, and a specificity of $90 \%$. Gouhar $\boldsymbol{e t}$ al. ${ }^{(10)}$ found that the ADC generated from DW-MRI as a non-invasive technique could differentiate tumor recurrence from radionecrosis in laryngeal carcinoma. Ohnishi et al. ${ }^{(3)}$ reported that a combination of a large tumor volume and a high ADC value could be predictive 
of local recurrence after definitive radiotherapy in hypopharyngeal or oropharyngeal squamous cell carcinoma. Vandecaveye $\boldsymbol{e t}$ al. ${ }^{(5)}$ stated that diffusionweighted imaging (DWI) with the ADC three weeks after the end of chemo-radiotherapy (CRT) for head and neck squamous cell carcinoma (HNSCC) allowed for early assessment of treatment response.

Tshering Vogel et al. ${ }^{(2)}$ stated that DW-MRI in combination with conventional MRI substantially improved the detection and exclusion of tumors in patients with laryngeal and hypopharyngeal cancers after treatment with (chemo) radiotherapy on both qualitative and quantitative analysis. Driessen et al. ${ }^{\text {(11) }}$ found that ADC was significantly correlated with cellularity, stromal component, and nuclearcytoplasmic ratio. The positive correlation of ADC and stromal component suggested that the poor prognostic value of high pretreatment $\mathrm{ADC}$ might partly be attributed to the tumor-stroma component, a known predictor of local failure. Acampora et al. ${ }^{(12)}$ stated that the ADC ratio had a promising value to differentiate between recurrent tumors and post-treatment changes in HNSCC and may be more useful than ADC1000 and ADC2000 with $82.0 \%$, Sensitivity, $100 \%$, specificity, and $90 \%$, accuracy of ADC ratio, by considering an optimal cutoff value of $65.5 \%$. Ailianou et al. ${ }^{(13)}$ stated that adding precise morphologic MRI criteria to quantitative DWI enables reproducible and accurate detection of recurrent head and neck squamous cell carcinoma/second primary head and neck squamous cell carcinoma occurring after treatment.

Khattab et al. ${ }^{(14)}$ stated that diffusion-weighted MRI could be a potential predictive biomarker for the therapeutic response of HNSCC to CRT. Primary tumors with higher pretreatment mean $\mathrm{ADC}$ and a smaller early into treatment percentage increase of mean ADC would be more likely to fail treatment. Serour $\boldsymbol{e t}$ al. ${ }^{(15)}$ found that incorporating the DWI sequence into the post-treatment imaging assessment protocol brought a substantial added value to conventional MRI assessment in patients with non-lymphoid head and neck malignancies with DW-MRI showing an accuracy of $90 \%$, a sensitivity of $88.9 \%$ and a specificity of 91.7\%. DW-MRI's valuable role could help avoid or, at least, largely minimize unnecessary or unfeasible tissue sampling. Zhang et al. ${ }^{(16)}$ stated an inverse correlation between ADC mean and metabolic tumor volume (MTV) in the HNSCC population and the correlativity depended on histological grading, clinical staging, and anatomical subsites of HNSCC. Freihat et al. ${ }^{(17)}$ stated that Diffusion-Weighted Imaging (DWI) derived from PET/MRI had a value in discriminating normal from metastatic lymph nodes with a significant difference between metastatic lymph nodes $<10 \mathrm{~mm}$ and the benign lymph nodes ADC mean. No significant difference between ADC means of the metastatic lymph nodes $<10 \mathrm{~mm}$ and the metastatic lymph nodes $>10$ $\mathrm{mm}$ and no significant differences between metastatic lymph nodes ADC mean values and different primary tumor grades or different primary tumor localization.

DW-MRI's advantages in head and neck lesions included being a non-invasive imaging modality for early detection of microstructural and functional changes in several pathologies with characterization between benign and inflammatory lesions. It allows early intra-treatment assessment in chemo-radiotherapy cases as it can identify some responding tumors before there is a change in size. It also allows post-treatment assessment and tumor recurrence ${ }^{(\mathbf{1 8 )}}$.

The limitations of the study included a small sample size and that diffusion-weighted imaging requires certain skill acquisition to be performed and interpreted correctly. Generally, MRI cannot be performed in patients with pacemakers or prosthetic valves. Another drawback of MRI is the long examination time (30-40min) and the necessity for patient compliance. Another limitation is the lack of correlation between ADC and pathological characteristics of positive biopsies for malignancy. We recommend further studies to evaluate such correlation.

\section{CONCLUSION}

DW-MRI is currently considered a diagnostic tool in various body cancers, especially in the head and neck, with the ability to detect recurrence after treatment. It is also used increasingly as a biomarker for early prediction of treatment response to chemo-radiotherapy (CRT). In our study, Diffusion-Weighted MRI had a $100 \%$ sensitivity, $80 \%$ specificity with an accuracy of $86.7 \%$ in comparison to histopathological findings of biopsies of the studied group, so we concluded that DWMRI had a diagnostic role in the detection of laryngeal and hypopharyngeal cancers in new, and the exclusion of tumor recurrence after chemo-radiotherapy in cases with suspicious lesions after completion of treatment.

\section{ABBREVIATIONS}

- ADC : Apparent diffusion coefficient

- a.u.: arbitrary unit

- DW-MRI: Diffusion Weighted Magnetic Resonance Imaging

- DWI: Diffusion Weighted Imaging

- MRI: Magnetic Resonance Imaging

- MTV: Metabolic tumor volume

- CRT: Chemoradiotherapy

- SPSS: Statistical Package for Social Science

- HNSCC: Head and neck squamous cell carcinoma.

\section{REFERENCES}

1. Thoeny H (2011): Diffusion-weighted MRI in head and neck radiology: applications in oncology. Cancer Imaging, 10 (1):209-14.

2. Tshering Vogel D, Zbaeren P, Geretschlaeger A et al. (2013): Diffusion-weighted MR imaging including bi- 
exponential fitting for the detection of recurrent or residual tumour after (chemo): radiotherapy for laryngeal and hypopharyngeal cancers. Eur Radiol., 23(2):562-9.

3. Ohnishi K, Shioyama Y, Hatakenaka M et al. (2011): Prediction of local failures with a combination of pretreatment tumor volume and apparent diffusion coefficient in patients treated with definitive radiotherapy for hypopharyngeal or oropharyngeal squamous cell carcinoma. J Radiat Res., 52 (4):522-30

4. Wang J, Takashima S, Takayama F et al. (2001): Head and neck lesions: characterization with diffusionweighted echo-planar MR imaging. Radiology, 220(3):621-30.

5. Vandecaveye V, Dirix P, De Keyzer F et al. (2012): Diffusion-weighted magnetic resonance imaging early after chemo-radiotherapy to monitor treatment response in head-and-neck squamous cell carcinoma. Int J Radiat Oncol Biol Phys., 82(3):1098-107

6. Brouwer J, Bodar E, de Bree $\mathbf{R}$ et al. (2004): Detecting recurrent laryngeal carcinoma after radiotherapy: room for improvement. European Archives of Oto-Rhino-Laryngology and Head \& Neck, 261(8): 417-422.

7. Vandecaveye V, De Keyzer F, Nuyts S et al. (2007): Detection of head and neck squamous cell carcinoma with diffusion weighted MRI after (chemo): radiotherapy: correlation between radiologic and histopathologic findings. Int J Radiat Oncol Biol Phys., 67(4):960-71.

8. Vandecaveye V, de Keyzer F, Vander Poorten V et al. (2006): Evaluation of the larynx for tumour recurrence by diffusion-weighted MRI after radiotherapy: initial experience in four cases. $\mathrm{Br} \mathrm{J}$ Radiol., 79(944):681-7.

9. Abdel Razek A, Kandeel A, Soliman N et al. (2007): Role of diffusion-weighted echo-planar MR imaging in differentiation of residual or recurrent head and neck tumors and post-treatment changes. AJNR Am J Neuroradiol., 28(6):1146-52.

10. Gouhar G, El-Hariri M (2011): Feasibility of diffusion weighted MR imaging in differentiating recurrent laryngeal carcinoma from radionecrosis. The Egyptian Journal of Radiology and Nuclear Medicine, 42 (2):169-175.

11. Driessen J, Caldas-Magalhaes J, Janssen $\mathbf{L}$ et al. (2014): Diffusion-weighted MR imaging in laryngeal and hypopharyngeal carcinoma: association between apparent diffusion coefficient and histologic findings. Radiology, 272(2):456-63.

12. Acampora A, Manzo G, Fenza G et al. (2016): High b-Value Diffusion MRI to Differentiate Recurrent Tumors from Post-treatment Changes in Head and Neck Squamous Cell Carcinoma: A Single Center Prospective Study. Biomed Res Int., 2016: 2865169.

13. Ailianou A, Mundada P, De Perrot T et al. (2018): MRI with DWI for the Detection of Posttreatment Head and Neck Squamous Cell Carcinoma: Why Morphologic MRI Criteria Matter. AJNR Am J Neuroradiol., 39(4):748-755.

14. Khattab H, Montasser M, Eid M et al. (2020): Diffusion-weighted magnetic resonance imaging (DWMRI): of head and neck squamous cell carcinoma: could it be an imaging biomarker for prediction of response to chemoradiation therapy. Egypt J Radiol Nucl Med., 51:204.

15. Serour D, Adel K, Osman A (2020): Post-treatment benign changes versus recurrence in non-lymphoid head and neck malignancies: can diffusion-weighted magnetic resonance imaging end up the diagnostic challenge? Egypt J Radiol Nucl Med., 51: 56-59.

16. Zhang L, Song $\mathbf{T}$, Meng $\mathbf{Z}$ et al. (2020): Correlation between apparent diffusion coefficients and metabolic parameters in hypopharyngeal squamous cell carcinoma: A prospective study with integrated PET/MRI. Eur J Radiol., 129:109070-5.

17. Freihat O, Pinter T, Kedves A et al. (2020): DiffusionWeighted Imaging (DWI): derived from PET/MRI for lymph node assessment in patients with Head and Neck Squamous Cell Carcinoma (HNSCC). Cancer Imaging, 20(1):56-65.

18. Dai Y, King A (2018): State of the art MRI in head and neck cancer. Clin Radiol., 73(1):45-59. 